Andreea Fulga*

\title{
A fixed point theorem involving rational expressions without using Picard iteration
}

https://doi.org/10.1515/taa-2020-0104

Received 18 May, 2021; accepted 1 August, 2021

Abstract: In this paper, we consider a certain fixed point theorem that contains some rational expressions. The main aim of this paper is to prove a fixed point theorem without using the Picard iteration.

Keywords: Picard Iteration, fixed point

MSC: 47H10,54H25,46J10, 46J15

\section{Introduction and Preliminaries}

Let T be a self-mapping on a Banach space $(X,\|\cdot\|)$ and $x_{0} \in X$. The sequence $\left\{x_{p}\right\}$ is called Picard sequence if $x_{p}=\mathrm{T} x_{p-1}$ for $n=1,2, \cdots$. In this case, the mapping $\mathrm{T}$ is said to be Picard operator.

In [1], the authors set out a different general principle for obtaining fixed points. We recall the main results of their paper.

Theorem 1. [1] Let $\mathrm{T}$ be self-mapping on a nonempty closed convex subset $\mathrm{C}$ of a Banach space $X$. If for some $\mathrm{x}_{0} \in \mathrm{C}$ there exists a constant $c, 0 \leq c<1$ such that

$$
\left\|\mathrm{x}_{p+2}-\mathrm{x}_{p+1}\right\| \leq c\left\|\mathrm{x}_{p+1}-\mathrm{x}_{p}\right\| \text { for all } p=0,1,2, \cdots,
$$

where

$$
\mathrm{x}_{p+1}:=\frac{1}{2}\left(\mathrm{x}_{p}+\mathrm{T} \mathrm{x}_{p}\right)
$$

Then, $\left\{x_{p}\right\}$ converges to a point $u$ in C. If, in addition, there exist the nonnegative constants $\alpha, \beta, \gamma, \delta, 0 \leq \gamma<1$ such that

$$
\left\|T x_{p}-T u\right\| \leq \alpha\left\|x_{p}-u\right\|+\beta\left\|x_{p}-T x_{p}\right\|+\gamma \max \left\{\|u-T u\|,\left\|x_{p}-T u\right\|, \delta\left\|u-T x_{p}\right\|\right\}
$$

for all $p$ sufficiently large, then $u$ is a fixed point of $T$.

A mapping $\varphi:[0, \infty) \rightarrow[0, \infty)$ is called a comparison function if it is increasing and $\varphi^{n}(t) \rightarrow 0, n \rightarrow \infty$, for any $t \in[0, \infty)$. We denote by $\Phi$, the class of the corporation function $\varphi:[0, \infty) \rightarrow[0, \infty)$. For more details and examples, see e.g. [2]-[6]. Among them, we recall the following essential result.

Lemma 2. (Berinde [2], Rus [3]) If $\varphi:[0, \infty) \rightarrow[0, \infty)$ is a comparison function, then:

(1) each iterate $\varphi^{k}$ of $\varphi, k \geq 1$, is also a comparison function;

(2) $\varphi$ is continuous at 0 ;

(3) $\varphi(t)<t$, for any $t>0$.

Later, Berinde [2] introduced the concept of (c)-comparison function in the following way.

^Corresponding Author: Andreea Fulga: Department of Mathematics and Computer Sciences, Transilvania University of Brasov, 500091, Brasov, Romania , E-mail: afulga@unitbv.ro 
Definition 3. (Berinde [2]) A function $\varphi:[0, \infty) \rightarrow[0, \infty)$ is said to be a (c)-comparison function if $\left(c_{1}\right) \varphi$ is monotone increasing,

$\left(c_{2}\right)$ the series $\sum_{j=1}^{\infty} \varphi^{j}(t)$ is convergent for any $t \in[0, \infty)$ (here $\varphi^{j}$ denotes the $j$-th iteration of $\varphi$ ).

By $\Psi$ we will denote the set of all (c)-comparison functions.

Very recently, Karapınar ([7]) announced the following interesting result.

Theorem 4. [7] On a nonempty closed convex subset $\mathrm{C}$ of a Banach space $X$, let $\mathrm{T}$ be a self-mapping. If for some $\mathrm{x}_{0} \in C$, there exists $a \varphi \in \Psi$ such that

$$
\left\|\mathrm{x}_{p+2}-\mathrm{x}_{p+1}\right\| \leq \varphi\left(\left\|\mathrm{x}_{p+1}-\mathrm{x}_{p}\right\|\right) \text { for all } p=0,1,2, \cdots,
$$

where

$$
\mathrm{x}_{p+1}:=\frac{1}{2}\left(\mathrm{x}_{p}+\mathrm{Tx}_{p}\right) .
$$

Then, $\left\{\mathrm{x}_{p}\right\}$ converges to a point $\mathrm{u}$ in $\mathrm{C}$. If, in addition, there exist the nonnegative constants $\alpha, \beta, \gamma, \delta, 0 \leq \gamma<1$, such that

$$
\left\|\mathrm{Tx}_{p}-\mathrm{T} u\right\| \leq \alpha\left\|\mathrm{x}_{p}-u\right\|+\beta\left\|\mathrm{x}_{p}-\mathrm{Tx}_{p}\right\|+\gamma \max \left\{\|u-\mathrm{T} u\|,\left\|\mathrm{x}_{-} \mathrm{T} u\right\|, \delta\left\|u-\mathrm{Tx}_{p}\right\|\right\},
$$

for all $p$ sufficiently large, then $u$ is a fixed point of $\mathrm{T}$.

\section{Main results}

First, we demonstrate the following useful Lemma:

Lemma 5. Let $\mathrm{C}$ be a nonempty closed convex subset of a Banach space $x$ and the sequence $\left\{x_{p}\right\}$ in $\mathrm{C}$. If for some $\mathrm{x}_{0} \in C$ there exists a function $\varphi \in \Psi$ such that

$$
\left\|\mathrm{x}_{p+2}-\mathrm{x}_{p+1}\right\| \leq \varphi\left(\left\|\mathrm{x}_{p+1}-\mathrm{x}_{p}\right\|\right) \text { for all } p=0,1,2, \cdots,
$$

then the sequence $\left\{\mathrm{x}_{p}\right\}$ converges to a point $u \in \mathrm{C}$.

Proof. Let $x_{0} \in C$ be such that the inequality (2.1) holds. Thus, recursively, from the inequality (2.1) and taking into account the monotony of the function $\varphi$, it follows that

$$
\left\|x_{p+1}-x_{p}\right\| \leq \varphi^{p}\left(\left\|\mathrm{x}_{1}-\mathrm{x}_{0}\right\|\right) \text { for all } p=0,1,2, \cdots
$$

and by $\left(c_{2}\right)$, we have that $\lim _{p \rightarrow \infty}\left\|\mathrm{x}_{p+1}-\mathrm{x}_{p}\right\|=0$, so the sequence $\left\{\mathrm{x}_{p}\right\}$ is asymptotically regular. Moreover, by the triangle inequality and taking $\left(c_{2}\right)$ into account, we get

$$
\begin{aligned}
\left\|\mathrm{x}_{p+n}-\mathrm{x}_{p}\right\| & \leq \sum_{j=p}^{p+n-1}\left\|\mathrm{x}_{j+1}-\mathrm{x}_{j}\right\| \leq \sum_{j=p}^{p+n-1} \varphi^{j}\left(\left\|\mathrm{x}_{1}-\mathrm{x}_{0}\right\|\right) \\
& =U_{n+p-1}-U_{p-1} \rightarrow 0 \text { as } p \rightarrow \infty,
\end{aligned}
$$

where $U_{p}=\sum_{j=n}^{p} \varphi^{j}\left(\left\|\mathrm{x}_{1}-\mathrm{x}_{0}\right\|\right)$. Thereupon, $\left\{\mathrm{x}_{p}\right\}$ is a Cauchy sequence and since, the subset $\mathrm{C}$ is closed, we deduce that $\left\{\mathrm{x}_{p}\right\}$ converges to a point in $u \in \mathrm{C}$.

Theorem 6. Let $\mathrm{C}$ be a nonempty closed convex subset of a Banach space $\mathrm{X}$, $\mathrm{T}$ be a self-mapping on $\mathrm{C}, \mathrm{x}_{0}$ be arbitrary in $\mathrm{C}$ and the sequence $\left\{\mathrm{x}_{n}\right\}$ in $\mathrm{C}$ defined as

$$
\mathrm{x}_{p+1}:=\frac{1}{2}\left(\mathrm{x}_{p}+\mathrm{T} \mathrm{x}_{p}\right) .
$$


Then the sequence $\left\{x_{p}\right\}$ is convergent provided that there exists $\varphi \in \Phi$ such that

$$
\left\|x_{p+2}-x_{p+1}\right\| \leq \varphi\left(\left\|x_{p+1}-x_{p}\right\|\right) \text { for all } p=0,1,2, \cdots \text {. }
$$

Moreover if $u=\lim _{p \rightarrow \infty} \mathrm{x}_{p}$ and there exist the constants $c_{1}, c_{2} \geq 0,0 \leq \lambda<1$, such that

$$
\begin{aligned}
\left\|\mathrm{Tx}_{p}-\mathrm{T} u\right\| & \leq c_{1}\left\|\mathrm{x}_{p}-u\right\|+c_{2} \max \left\{\frac{\left.\left\|\mathrm{x}_{p}-\mathrm{Tx}_{p}\right\|(1+\|u-\mathrm{T} u\|)\right)}{1+\left\|\mathrm{x}_{p}-u\right\|},\left\|u-\mathrm{Tx}_{p}\right\|\right\} \\
& +\lambda \max \left\{\frac{\|u-\mathrm{T} u\|\left(1+\left\|\mathrm{x}_{p}-\mathrm{Tx}\right\|\right)}{1+\left\|\mathrm{x}_{p}-u\right\|},\left\|\mathrm{x}_{p}-\mathrm{T} u\right\|\right\}
\end{aligned}
$$

for $p$ sufficiently large, then $u$ is a fixed point of $\mathrm{T}$.

Proof. Let $\mathrm{x}_{0} \in \mathrm{C}$ and the sequence $\left\{\mathrm{x}_{p}\right\}$ be defined by (2.4). Thus, keeping in mind (2.10), by Lemma 5 it follows that $\left\{x_{p}\right\}$ is a convergent sequence. Let $u \in C$ be the limit of $x_{p}$. We claim that $u$ is a fixed point of the mapping $\mathrm{T}$. Indeed, we can easily see that (2.4) can be rewritten as $\mathrm{Tx}_{p}-\mathrm{x}_{p}=2\left(\mathrm{x}_{p+1}-\mathrm{x}_{p}\right)$. Therefore,

$$
\left\|\mathrm{x}_{p}-\mathrm{Tx}_{p}\right\|=2\left\|\mathrm{x}_{p+1}-\mathrm{x}_{p}\right\| \leq 2 \varphi^{p}\left(\left\|\mathrm{x}_{1}-\mathrm{x}_{0}\right\|\right) \rightarrow 0 \text { as } p \rightarrow \infty
$$

But, since $\lim _{p \rightarrow \infty} x_{p}=u$, taking into account the uniqueness of the limit we get that $\lim _{p \rightarrow \infty} \operatorname{Tx}_{p}=u$. Now, letting the limit of (2.11) as $p \rightarrow \infty$, we have

$$
\begin{aligned}
\|u-\mathrm{T} u\|= & \lim _{p \rightarrow \infty}\left\|\mathrm{Tx}_{p}-\mathrm{T} u\right\| \\
\leq & \lim _{p \rightarrow \infty}\left[c_{1}\left\|\mathrm{x}_{p}-u\right\|+c_{2} \max \left\{\left\|u-\mathrm{Tx}_{p}\right\|, \frac{\left\|\mathrm{x}_{p}-\mathrm{Tx}_{p}\right\|(1+\|u-\mathrm{T} u\|)}{1+\left\|\mathrm{x}_{p}-u\right\|}\right\}+\right. \\
& \left.+\lambda \max \left\{\left\|\mathrm{x}_{p}-\mathrm{T} u\right\|, \frac{\|u-\mathrm{T} u\|\left(1+\left\|\mathrm{x}_{p}-\mathrm{Tx}_{p}\right\|\right)}{1+\left\|\mathrm{x}_{p}-u\right\|}\right\}\right] \\
= & \lambda\|u-\mathrm{T} u\|,
\end{aligned}
$$

which is a contradiction. Thereupon, $u=\mathrm{T} u$.

Adding a supplementary condition in Theorem 6, we can assure the uniqueness of the fixed point.

Theorem 7. If in Theorem 6 the constants $c_{1}, c_{2}, \lambda \geq$ are such that $c_{1}+c_{2}+\lambda<1$ then the fixed point of the mapping $\mathrm{T}$ is unique.

Proof. Let $\left\{\mathrm{x}_{p}\right\}$ be the sequence defined by (2.4). We know by the previous proof, that $\left\{\mathrm{x}_{p}\right\}$ is convergent to a point $u \in \mathrm{C}$. More than that,

$$
\lim _{p \rightarrow \infty} \mathrm{x}_{p}=\lim _{p \rightarrow \infty} \mathrm{Tx}_{p}=u \text { and } \mathrm{T} u=u .
$$

Supposing than there exists a point $v \in \mathrm{C}$ such that $\mathrm{T} v=v \neq u$, by (2.11) we have

$$
\begin{gathered}
\left\|\mathrm{Tx}_{p}-v\right\|=\left\|\mathrm{T} \mathrm{x}_{p}-\mathrm{T} v\right\| \leq c_{1}\left\|\mathrm{x}_{p}-v\right\|+c_{2} \max \left\{\frac{\left.\left\|\mathrm{x}_{p}-\mathrm{Tx}_{p}\right\|(1+\|v-\mathrm{T} v\|)\right)}{1+\left\|\mathrm{x}_{p}-v\right\|},\left\|v-\mathrm{Tx}_{p}\right\|\right\} \\
+\lambda \max \left\{\frac{\|v-\mathrm{T} v\|\left(1+\left\|\mathrm{x}_{p}-\mathrm{T} \mathrm{x}_{p}\right\|\right)}{1+\left\|\mathrm{x}_{p}-v\right\|},\left\|\mathrm{x}_{p}-\mathrm{T} v\right\|\right\} \\
=c_{1}\left\|\mathrm{x}_{p}-v\right\|+c_{2} \max \left\{\frac{\left.\left\|\mathrm{x}_{p}-\mathrm{T} \mathrm{x}_{p}\right\|(1+\|v-v\|)\right)}{1+\left\|\mathrm{x}_{p}-v\right\|},\left\|v-\mathrm{Tx}_{p}\right\|\right\} \\
+\lambda \max \left\{\frac{\|v-v\|\left(1+\left\|\mathrm{x}_{p}-\mathrm{T} \mathrm{x}_{p}\right\|\right)}{1+\left\|\mathrm{x}_{p}-v\right\|},\left\|\mathrm{x}_{p}-v\right\|\right\} \\
=c_{1}\left\|\mathrm{x}_{p}-v\right\|+c_{2} \max \left\{\frac{\left\|\mathrm{x}_{p}-\mathrm{Tx}_{p}\right\|}{1+\left\|\mathrm{x}_{p}-v\right\|},\left\|v-\mathrm{Tx}_{p}\right\|\right\} \\
+\lambda \max \left\{0,\left\|\mathrm{x}_{p}-v\right\|\right\} .
\end{gathered}
$$


Taking the limit as $p \rightarrow \infty$ in (2.8) we get

$$
\|u-v\| \leq c_{1}\|u-v\|+c_{2}\|u-v\|+\lambda\|u-v\|=\left(c_{1}+c_{2}+\lambda\right)\|u-v\|<\|u-v\| .
$$

This is a contradiction, so that $u=v$.

Corollary 8. Let $\mathrm{C}$ be a nonempty closed convex subset of a Banach space $x, \mathrm{~T}$ be a self-mapping on $\mathrm{C}, \mathrm{x}_{0}$ be arbitrary in $\mathrm{C}$ and the sequence $\left\{\mathrm{x}_{n}\right\}$ in $\mathrm{C}$ defined as

$$
\mathrm{x}_{p+1}:=\frac{1}{2}\left(\mathrm{x}_{p}+\mathrm{Tx}_{p}\right)
$$

Then the sequence $\left\{\mathrm{x}_{p}\right\}$ is convergent provided that there exists $0 \leq \kappa<1$ such that

$$
\left\|x_{p+2}-x_{p+1}\right\| \leq \kappa \cdot\left\|\mathrm{x}_{p+1}-\mathrm{x}_{p}\right\| \text { for all } p=0,1,2, \cdots .
$$

Moreover if $u=\lim _{p \rightarrow \infty} x_{p}$ and there exist the constants $c_{1}, c_{2} \geq 0,0 \leq \lambda<1$, such that

$$
\begin{aligned}
\left\|\mathrm{Tx}_{p}-\mathrm{T} u\right\| & \leq c_{1}\left\|\mathrm{x}_{p}-u\right\|+c_{2} \max \left\{\frac{\left.\left\|\mathrm{x}_{p}-\mathrm{Tx}_{p}\right\|(1+\|u-\mathrm{T} u\|)\right)}{1+\left\|\mathrm{x}_{p}-u\right\|},\left\|u-T x_{n}\right\|\right\} \\
& +\lambda \max \left\{\frac{\|u-\mathrm{T} u\|\left(1+\left\|\mathrm{x}_{p}-\mathrm{Tx}_{p}\right\|\right)}{1+\left\|\mathrm{x}_{p}-u\right\|},\left\|x_{n}-\mathrm{T} u\right\|\right\}
\end{aligned}
$$

for all $p$ sufficiently large, then $u$ is a fixed point of $\mathrm{T}$.

Proof. Put $\varphi(t)=\kappa t$, with $0 \leq \kappa<1$, in Theorem 6 .

Corollary 9. Let $\mathrm{C}$ be a nonempty closed convex subset of a Banach space $x$, and $\mathrm{T}, \mathrm{G}: \mathrm{C} \rightarrow \mathrm{C}$ be two mappings such that $\mathrm{Gx}=\frac{\mathrm{x}+\mathrm{Tx}}{2}$, for any $\mathrm{x} \in \mathrm{C}$. Supposing that there exists $0<\Upsilon<2$ such that

$$
\|\mathrm{Tx}-\mathrm{Ty}\| \leq \Upsilon \max \left\{\begin{array}{c}
\|\mathrm{x}-\mathrm{y}\|, \frac{1}{2} \frac{\|\mathrm{x}-\mathrm{Tx}\|(1+\|\mathrm{y}-\mathrm{Ty}\|)}{1+\|\mathrm{x}-\mathrm{y}\|+\|\mathrm{x}-\mathrm{Ty}\|},\|\mathrm{y}-\mathrm{Tx}\|, \\
\frac{1}{4} \frac{\|\mathrm{y}-\mathrm{Ty}\|(1+\|\mathrm{x}-\mathrm{Tx}\|)}{1+\|\mathrm{x}-\mathrm{y}\|}, \frac{1}{2}\|\mathrm{x}-\mathrm{Ty}\|
\end{array}\right\},
$$

for any $\mathrm{x}, \mathrm{y} \in \mathrm{C}$. If there exist two positive real numbers $a, b$, with $a<\frac{2}{\Upsilon}$ and $b<1$ such that

$$
\begin{aligned}
& \|\mathrm{x}-\mathrm{TG}\| \leq a\left\|\mathrm{~T}^{2} \mathrm{x}-\mathrm{TG}\right\|, \text { for any } \mathrm{x} \in \mathrm{C} ; \\
& \left\|\mathrm{T}^{2} \mathrm{x}-\omega\right\| \leq b\|\mathrm{x}-\omega\|, \text { for } \omega \in\{\mathrm{Tx}, \mathrm{Gx}\},
\end{aligned}
$$

then there exists at least one point $u \in \mathrm{C}$ such that $\mathrm{T} u=u$.

Proof. First of all, by considering the definition of the mapping G, we can easily obtain the following relations:

$$
\begin{aligned}
& \|x-T x\|=\|x-2 G x+x\|=2\|G x-x\|, \\
& \|G x-T x\|=\|G x-2 G x+x\|=\|G x-x\|, \\
& \|G x-T G x\|=\left\|G x-2 G^{2} x+G x\right\|=2\left\|G^{2} x-G x\right\| .
\end{aligned}
$$

Let $\mathrm{x}_{0}$ be an arbitrary but fixed point in $\mathrm{C}$ and the sequence $\left\{\mathrm{x}_{p}\right\}$ defined as follows:

$$
\mathrm{x}_{1}=\mathrm{Gx}_{0}, \ldots, \mathrm{x}_{p}=\mathrm{Gx}_{p-1}=\mathrm{G}^{p} \mathrm{x}_{0} .
$$

Using this notation, the relations (2.14) become

$$
\begin{aligned}
& \left\|\mathrm{x}_{p}-\mathrm{Tx}_{p}\right\|=2\left\|\mathrm{x}_{p}-\mathrm{x}_{p+1}\right\|, \\
& \left\|\mathrm{x}_{p+1}-\mathrm{Tx}_{p}\right\|=\left\|\mathrm{x}_{p+1}-\mathrm{x}_{p}\right\|, \\
& \left\|\mathrm{x}_{p+1}-\mathrm{Tx}_{p+1}\right\|=2\left\|\mathrm{x}_{p+2}-\mathrm{x}_{p+1}\right\| .
\end{aligned}
$$


Thus,

$$
\begin{aligned}
\left\|\mathrm{x}_{p+2}-\mathrm{x}_{p+1}\right\| & =\frac{1}{2}\left\|\mathrm{x}_{p+1}-\mathrm{Tx}_{p+1}\right\|=\frac{1}{4}\left\|2 \mathrm{x}_{p+1}-2 \mathrm{Tx}_{p+1}\right\|=\frac{1}{4}\left\|\left(2 \mathrm{x}_{p+1}-\mathrm{Tx}_{p+1}\right)-\mathrm{Tx}_{p+1}\right\| \\
& \leq \frac{1}{4}\left[\left\|2 \mathrm{x}_{p+1}-\mathrm{Tx}_{p+1}-\mathrm{Tx}_{p}\right\|+\left\|\mathrm{Tx}_{p}-\mathrm{Tx}_{p+1}\right\|\right] \\
& =\frac{1}{4}\left[\left\|\mathrm{x}_{p}+\mathrm{Tx}_{p}-\mathrm{Tx}_{p+1}-\mathrm{Tx}_{p}\right\|+\left\|\mathrm{Tx}_{p}-\mathrm{Tx}_{p+1}\right\|\right] \\
& =\frac{1}{4}\left[\left\|\mathrm{x}_{p}-\mathrm{Tx}_{p+1}\right\|+\left\|\mathrm{Tx}_{p}-\mathrm{Tx}_{p+1}\right\|\right] \\
& \leq \frac{1}{2} \max \left\{\left\|\mathrm{x}_{p}-\mathrm{Tx}_{p+1}\right\|,\left\|\mathrm{Tx}_{p}-\mathrm{Tx}_{p+1}\right\|\right\}
\end{aligned}
$$

In case that $\max \left\{\left\|\mathrm{x}_{p}-\mathrm{Tx}_{p+1}\right\|,\left\|\mathrm{Tx}_{p}-\mathrm{Tx}_{p+1}\right\|\right\}=\left\|\mathrm{Tx}_{p}-\mathrm{Tx} \mathrm{x}_{p+1}\right\|$, by (2.12) and keeping in mind (2.16), we obtain

$$
\begin{aligned}
& \left\|\mathrm{x}_{p+2}-\mathrm{x}_{p+1}\right\| \leq \frac{1}{2}\left\|\mathrm{Tx}_{p}-\mathrm{Tx}_{p+1}\right\| \\
& \leq \frac{\Upsilon}{2} \max \left\{\begin{array}{c}
\left\|\mathrm{x}_{p}-\mathrm{x}_{p+1}\right\|, \frac{1}{2} \frac{\left\|\mathrm{x}_{p}-\mathrm{Tx}_{p}\right\|\left(1+\left\|\mathrm{x}_{p+1}-\mathrm{Tx}_{p+1}\right\|\right)}{1+\left\|\mathrm{x}_{p}-\mathrm{x}_{p+1}\right\|+\left\|\mathrm{x}_{p}-\mathrm{Tx}_{p+1}\right\|},\left\|\mathrm{x}_{p+1}-\mathrm{T} \mathrm{x}_{p}\right\|, \\
\frac{1}{4} \frac{\left\|\mathrm{x}_{p+1}-\mathrm{Tx}_{p+1}\right\|\left(1+\left\|\mathrm{x}_{p}-\mathrm{Tx}_{p}\right\|\right)}{1+\left\|\mathrm{x}_{p}-\mathrm{x}_{p+1}\right\|}, \frac{1}{2}\left\|\mathrm{x}_{p}-\mathrm{Tx}_{p+1}\right\|
\end{array}\right\} \\
& \leq \frac{1}{2} \max \left\{\begin{array}{c}
\left\|\mathrm{x}_{p}-\mathrm{x}_{p+1}\right\|, \frac{1}{2} \frac{2\left\|\mathrm{x}_{p}-\mathrm{x}_{p+1}\right\|\left(1+\left\|\mathrm{x}_{p+1}-\mathrm{x}_{p}\right\|+\left\|\mathrm{x}_{p}-\mathrm{Tx}_{p+1}\right\|\right)}{1+\left\|\mathrm{x}_{p}-\mathrm{x}_{p+1}\right\|+\left\|\mathrm{x}_{p}-T \mathrm{x}_{p+1}\right\|},\left\|\mathrm{x}_{p+1}-\mathrm{x}_{p}\right\|, \\
\frac{1}{4} \frac{2\left\|\mathrm{x}_{p+1}-\mathrm{x}_{p+2}\right\|\left(1+\left\|\mathrm{x}_{p}-\mathrm{x}_{p+1}\right\|\right)}{1+\left\|\mathrm{x}_{p}-\mathrm{x}_{p+1}\right\|}, \frac{1}{2}\left[\left\|\mathrm{x}_{p}-\mathrm{x}_{p+1}\right\|+\left\|\mathrm{x}_{p+1}-\mathrm{Tx}_{p+1}\right\|\right.
\end{array}\right\} \\
& \leq \frac{r}{2} \max \left\{\begin{array}{c}
\left\|\mathbf{x}_{p}-\mathbf{x}_{p+1}\right\|,\left\|\mathbf{x}_{p}-\mathbf{x}_{p+1}\right\|,\left\|\mathbf{x}_{p+1}-\mathbf{x}_{p}\right\|, \\
\frac{1}{2}\left\|\mathbf{x}_{p+1}-\mathbf{x}_{p+2}\right\|, \frac{1}{4}\left[\left\|\mathbf{x}_{p}-\mathbf{x}_{p+1}\right\|+2\left\|\mathbf{x}_{p+1}-\mathbf{x}_{p+2}\right\|\right.
\end{array}\right\} \\
& \leq \frac{\Upsilon}{2} \max \left\{\begin{array}{c}
\left\|\mathbf{x}_{p}-\mathbf{x}_{p+1}\right\|,\left\|\mathbf{x}_{p}-\mathbf{x}_{p+1}\right\|,\left\|\mathbf{x}_{p+1}-\mathbf{x}_{p}\right\|, \\
\frac{1}{2}\left\|\mathbf{x}_{p+1}-\mathbf{x}_{p+2}\right\|, \frac{1}{2} \max \left\{\left\|\mathbf{x}_{p}-\mathbf{x}_{p+1}\right\|, 2\left\|\mathbf{x}_{p+1}-\mathbf{x}_{p+2}\right\|\right\}
\end{array}\right\} \\
& \leq \frac{\Upsilon}{2} \max \left\{\left\|\mathrm{x}_{p}-\mathrm{x}_{p+1}\right\|,\left\|\mathrm{x}_{p+1}-\mathrm{x}_{p+2}\right\|\right\} .
\end{aligned}
$$

Of course, since in case that $\max \left\{\left\|\mathrm{x}_{p}-\mathrm{x}_{p+1}\right\|,\left\|\mathrm{x}_{p+1}-\mathrm{x}_{p+2}\right\|\right\}=\left\|\mathrm{x}_{p+1}, \mathrm{x}_{p+2}\right\|$, we get a contradiction, it follows that

$$
\left\|\mathrm{x}_{p+2}-\mathrm{x}_{p+1}\right\| \leq \frac{\Upsilon}{2}\left\|\mathrm{x}_{p+1}-\mathrm{x}_{p}\right\|
$$

for $p \in \mathbb{N}$.

In the second case, when $\max \left\{\left\|\mathrm{x}_{p}-\mathrm{Tx}_{p+1}\right\|,\left\|\mathrm{Tx}_{p}-\mathrm{Tx}_{p+1}\right\|\right\}=\left\|\mathrm{x}_{p}-\mathrm{Tx}_{p+1}\right\|$, we have

$$
\left\|\mathrm{x}_{p+2}-\mathrm{x}_{p+1}\right\| \leq \frac{1}{2}\left\|\mathrm{x}_{p}-\mathrm{Tx}_{p+1}\right\|
$$

But, from (2.13), we get

$$
\begin{aligned}
& \left\|\mathrm{x}_{p}-\mathrm{Tx}_{p+1}\right\| \leq a\left\|\mathrm{~T}^{2} \mathrm{x}_{p}-\mathrm{Tx}_{p+1}\right\|, \\
& \left\|\mathrm{T}^{2} \mathrm{x}_{p}-\mathrm{T} \mathrm{x}_{p}\right\| \leq b\left\|\mathrm{x}_{p}-\mathrm{T} \mathrm{x}_{p}\right\|=2 b\left\|\mathrm{x}_{p}-\mathrm{x}_{p+1}\right\|, \\
& \left\|\mathrm{T}^{2} \mathrm{x}_{p}-\mathrm{x}_{p+1}\right\| \leq b\left\|\mathrm{x}_{p}-\mathrm{x}_{p+1}\right\|,
\end{aligned}
$$


and then

$$
\begin{aligned}
& \left\|\mathrm{x}_{p+2}-\mathrm{x}_{p+1}\right\|=\frac{1}{2}\left\|\mathrm{x}_{p}-\mathrm{Tx}_{p+1}\right\| \leq \frac{a}{2}\left\|\mathrm{~T}^{2} \mathrm{x}_{p}-\mathrm{Tx}_{p+1}\right\|
\end{aligned}
$$

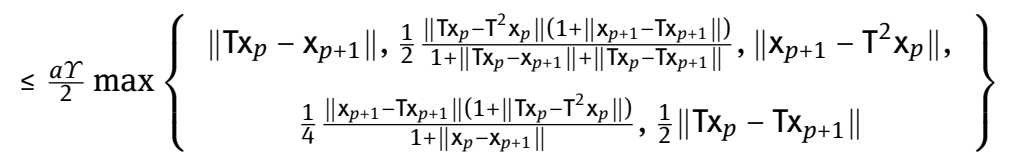

$$
\begin{aligned}
& =\frac{a \Upsilon}{2} \max \left\{\begin{array}{c}
\left\|\mathrm{x}_{p}-\mathrm{x}_{p+1}\right\|, \frac{1}{2} \frac{2 b\left\|\mathrm{x}_{p}-\mathrm{x}_{p+1}\right\|\left(1+\left\|\mathrm{x}_{p+1}-\mathrm{T} \mathrm{x}_{p}\right\|+\left\|\mathrm{T} \mathrm{T}_{p}-\mathrm{T} \mathrm{x}_{p+1}\right\|\right)}{1+\left\|\mathrm{T} \mathrm{T}_{p}-\mathrm{x}_{p+1}\right\|+\left\|\mathrm{T} \mathrm{x}_{p}-\mathrm{T} \mathrm{x}_{p+1}\right\|}, \\
b\left\|\mathrm{x}_{p+1}-\mathrm{x}_{p}\right\|, \frac{1}{4} \frac{2\left\|\mathrm{x}_{p+1}-\mathrm{x}_{p+2}\right\|\left(1+2 b\left\|\mathrm{x}_{p}-\mathrm{x}_{p+1}\right\|\right)}{1+\left\|\mathrm{x}_{p}-\mathrm{x}_{p+1}\right\|}, \\
\frac{r}{2} \max \left\{\left\|\mathrm{x}_{p}-\mathrm{x}_{p+1}\right\|,\left\|\mathrm{x}_{p+1}-\mathrm{x}_{p+2}\right\|\right\}
\end{array}\right\} \\
& \leq \frac{a \Upsilon}{2} \max \left\{\left\|\mathrm{x}_{p+1}-\mathrm{x}_{p}\right\|, b\left\|\mathrm{x}_{p+1}-\mathrm{x}_{p}\right\|,\left\|\mathrm{x}_{p+1}-\mathrm{x}_{p+2}\right\|\right\} \\
& =\frac{a \Upsilon}{2} \max \left\{\left\|\mathbf{x}_{p+1}-\mathbf{x}_{p}\right\|,\left\|\mathbf{x}_{p+1}-\mathbf{x}_{p+2}\right\|\right\} .
\end{aligned}
$$

Consequently, since $\frac{a \Upsilon}{2}<1$, we get

$$
\left\|\mathrm{x}_{p+1}-\mathrm{x}_{p+2}\right\| \leq \frac{a \Upsilon}{2} \mid \mathrm{x}_{p+1}-\mathrm{x}_{p} \| .
$$

Thereupon, if we denote $\kappa=\max \left\{\frac{\Upsilon}{2}, \frac{a \Upsilon}{2}\right\}$, from (2.18) and (2.18) we have

$$
\left\|\mathrm{x}_{p+1}-\mathrm{x}_{p+2}\right\| \leq \kappa \mid \mathrm{x}_{p+1}-\mathrm{x}_{p} \|,
$$

for any $p \in \mathbb{N}$.

On the other hand, from the inequality (2.12), for $\mathrm{x}=\mathrm{x}_{p}$ and $\mathrm{y}=u$, we get

$$
\begin{gathered}
\left\|\mathrm{Tx}_{p}-\mathrm{T} u\right\| \leq \Upsilon \max \left\{\begin{array}{c}
\left\|\mathrm{x}_{p}-u\right\|, \frac{1}{2} \frac{\left\|\mathrm{x}_{p}-\mathrm{T} \mathrm{x}_{p}\right\|(1+\|u-\mathrm{T} u\|)}{1+\left\|\mathrm{x}_{p}-u\right\|+\left\|\mathrm{x}_{p}-\mathrm{T} u\right\|},\left\|u-\mathrm{Tx}_{p}\right\|, \\
\frac{1}{4} \frac{\|u-\mathrm{T} u\|\left(1+\left\|\mathrm{x}_{p}-\mathrm{T} \mathrm{x}_{p}\right\|\right)}{1+\left\|\mathrm{x}_{p}-u\right\|}, \frac{1}{2}\left\|\mathrm{x}_{p}-\mathrm{T} u\right\|
\end{array}\right\} \\
\leq \Upsilon\left\|\mathrm{x}_{p}-u\right\|+\frac{\Upsilon}{2} \max \left\{\frac{\left\|\mathrm{x}_{p}-\mathrm{T} \mathrm{x}_{p}\right\|(1+\|u-\mathrm{T} u\|)}{1+\left\|\mathrm{x}_{p}-u\right\|+\left\|\mathrm{x}_{p}-\mathrm{T} u\right\|},\left\|u-\mathrm{Tx}_{p}\right\|\right\}+ \\
+\frac{\Upsilon}{4} \max \left\{\frac{\|u-\mathrm{T} u\|\left(1+\left\|\mathrm{x}_{p}-\mathrm{T} \mathrm{x}_{p}\right\|\right)}{1+\left\|\mathrm{x}_{p}-u\right\|},\left\|\mathrm{x}_{p}-\mathrm{T} u\right\|\right\} \\
\leq \Upsilon\left\|\mathrm{x}_{p}-u\right\|+\frac{\Upsilon}{2} \max \left\{\frac{\left\|\mathrm{x}_{p}-\mathrm{T} \mathrm{x}_{p}\right\|(1+\|u-\mathrm{T} u\|)}{1+\left\|\mathrm{x}_{p}-u\right\|},\left\|u-\mathrm{Tx}_{p}\right\|\right\}+ \\
+\frac{\Upsilon}{4} \max \left\{\frac{\|u-\mathrm{T} u\|\left(1+\left\|\mathrm{x}_{p}-\mathrm{T} \mathrm{x}_{p}\right\|\right)}{1+\left\|\mathrm{x}_{p}-u\right\|},\left\|\mathrm{x}_{p}-\mathrm{T} u\right\|\right\} .
\end{gathered}
$$

Moreover, choosing $c_{1}=\Upsilon, c_{2}=\frac{\Upsilon}{2}$ and $\lambda=\frac{\Upsilon}{4}$, for $p$ sufficiently large, we get (2.11) and then, by Theorem 6 it follows that $u$ is a fixed point of T.

Corollary 10. Let $\mathrm{C}$ be a nonempty closed convex subset of a Banach space $\mathrm{X}$, and $\mathrm{T}: \mathrm{C} \rightarrow \mathrm{C}$ be a mapping such that $\mathrm{T}^{2}=$ I. If there exists $0 \leq \Upsilon<2$ such that

$$
\|\mathrm{Tx}-\mathrm{Ty}\| \leq \Upsilon \max \left\{\begin{array}{c}
\|\mathrm{x}-\mathrm{y}\|, \frac{1}{2} \frac{\|\mathrm{x}-\mathrm{T} x\|(1+\|\mathrm{y}-\mathrm{T} \mathrm{y}\|)}{1+\|\mathrm{x}-\mathrm{y}\|},\|\mathrm{y}-\mathrm{Tx}\|, \\
\frac{1}{4} \frac{\|\mathrm{y}-\mathrm{Ty}\|(1+\|\mathrm{x}-\mathrm{Tx}\|)}{1+\|\mathrm{x}-\mathrm{y}\|}, \frac{1}{2}\|\mathrm{x}-\mathrm{Ty}\|
\end{array}\right\},
$$

for any $\mathrm{x}, \mathrm{y} \in \mathrm{C}$ then we can find at least one point $u \in \mathrm{C}$ such that $\mathrm{T} u=u$. 
Proof. Let $\mathrm{x}_{0}$ be an arbitrary but fixed point in $\mathrm{C}$ and the sequence $\left\{\mathrm{x}_{p}\right\}$ defined by (2.15). On the other hand, by (2.16) we have:

$$
\begin{aligned}
& \left\|\mathrm{x}_{p}-\mathrm{Tx}_{p}\right\|=2\left\|\mathrm{x}_{p}-\mathrm{x}_{p+1}\right\|, \\
& \left\|\mathrm{x}_{p+1}-\mathrm{Tx}_{p}\right\|=\left\|\mathrm{x}_{p+1}-\mathrm{x}_{p}\right\|, \\
& \left\|\mathrm{x}_{p+1}-\mathrm{Tx}_{p+1}\right\|=2\left\|\mathrm{x}_{p+2}-\mathrm{x}_{p+1}\right\| .
\end{aligned}
$$

Thus,

$$
\begin{aligned}
\left\|\mathrm{x}_{p+2}-\mathrm{x}_{p+1}\right\| & =\frac{1}{2}\left\|\mathrm{x}_{p+1}-\mathrm{Tx}_{p+1}\right\|=\frac{1}{4}\left\|2 \mathrm{x}_{p+1}-2 \mathrm{Tx}_{p+1}\right\|=\frac{1}{4}\left\|\left(2 \mathrm{x}_{p+1}-\mathrm{Tx}_{p+1}\right)-\mathrm{Tx}_{p+1}\right\| \\
& \leq \frac{1}{4}\left[\left\|2 \mathrm{x}_{p+1}-\mathrm{Tx}_{p+1}-\mathrm{Tx}_{p}\right\|+\left\|\mathrm{Tx}_{p}-\mathrm{Tx}_{p+1}\right\|\right] \\
& =\frac{1}{4}\left[\left\|\mathrm{x}_{p}+\mathrm{Tx}_{p}-\mathrm{Tx}_{p+1}-\mathrm{Tx}_{p}\right\|+\left\|\mathrm{Tx}_{p}-\mathrm{Tx}_{p+1}\right\|\right] \\
& =\frac{1}{4}\left[\left\|\mathrm{x}_{p}-\mathrm{Tx}_{p+1}\right\|+\left\|\mathrm{Tx}_{p}-\mathrm{Tx}_{p+1}\right\|\right] \\
& \leq \frac{1}{2} \max \left\{\left\|\mathrm{x}_{p}-\mathrm{Tx}_{p+1}\right\|,\left\|\mathrm{Tx}_{p}-\mathrm{Tx}_{p+1}\right\|\right\}
\end{aligned}
$$

and we distinguish two cases:

Case 1. $\max \left\{\left\|\mathrm{x}_{p}-\mathrm{Tx}_{p+1}\right\|,\left\|\mathrm{Tx}_{p}-\mathrm{Tx}_{p+1}\right\|\right\}=\left\|\mathrm{Tx}_{p}-\mathrm{Tx}_{p+1}\right\|$.

Then, by (2.22), keeping in mind (2.16) and following the same arguments as in Corollary 9 we get

$$
\begin{aligned}
\left\|\mathbf{x}_{p+2}-\mathbf{x}_{p+1}\right\| & \leq \frac{1}{2}\left\|\mathrm{Tx}_{p}-\mathrm{Tx}_{p+1}\right\| \\
& \leq \frac{\Upsilon}{2} \max \left\{\left\|\mathbf{x}_{p}-\mathbf{x}_{p+1}\right\|,\left\|\mathrm{x}_{p+1}-\mathbf{x}_{p+2}\right\|\right\} \\
& \leq \frac{\Upsilon}{2}\left\|\mathbf{x}_{p+1}-\mathbf{x}_{p}\right\|,
\end{aligned}
$$

for $p \in \mathbb{N}$ (since $\left.\frac{r}{2}<1\right)$.

Case 2. $\max \left\{\left\|\mathrm{x}_{p}-\mathrm{Tx}_{p+1}\right\|,\left\|\mathrm{Tx}_{p}-\mathrm{Tx}_{p+1}\right\|\right\}=\left\|\mathrm{x}_{p}-\mathrm{Tx}_{p+1}\right\|$. We have

$$
\begin{aligned}
& \left\|\mathrm{x}_{p+2}-\mathrm{x}_{p+1}\right\| \leq \frac{1}{2}\left\|\mathrm{x}_{p}-\mathrm{Tx}_{p+1}\right\|=\frac{1}{2}\left\|\mathrm{~T}^{2} \mathrm{x}_{p}-\mathrm{Tx}_{p+1}\right\| \\
& \leq \frac{r}{2} \max \left\{\begin{array}{c}
\left\|\mathrm{Tx}_{p}-\mathrm{x}_{p+1}\right\|, \frac{1}{2} \frac{\left\|\mathrm{Tx}_{p}-\mathrm{T}^{2} \mathrm{x}_{p}\right\|\left(1+\left\|\mathrm{x}_{p+1}-\mathrm{T} \mathrm{T}_{p+1}\right\|\right)}{1+\left\|\mathrm{T} \mathrm{T}_{p}-\mathrm{x}_{p+1}\right\|+\left\|\mathrm{T} \mathrm{T}_{p}-\mathrm{T} \mathrm{T}_{p+1}\right\|},\left\|\mathrm{x}_{p+1}-\mathrm{T}^{2} \mathrm{x}_{p}\right\|, \\
\frac{1}{4} \frac{\left\|\mathrm{x}_{p+1}-\mathrm{T} \mathrm{x}_{p+1}\right\|\left(1+\left\|\mathrm{T} \mathrm{x}_{p}-\mathrm{T}^{2} \mathrm{x}_{p}\right\|\right)}{1+\left\|\mathrm{x}_{p}-\mathrm{x}_{p+1}\right\|}, \frac{1}{2}\left\|\mathrm{~T} \mathrm{x}_{p}-\mathrm{T} \mathrm{T}_{p+1}\right\|
\end{array}\right\} \\
& =\frac{r}{2} \max \left\{\begin{array}{c}
\left\|\mathrm{x}_{p}-\mathrm{x}_{p+1}\right\|, \frac{1}{2} \frac{\left\|\mathrm{T} \mathrm{x}_{p}-\mathrm{x}_{p}\right\|\left(1+\left\|\mathrm{x}_{p+1}-\mathrm{T} \mathrm{x}_{p}\right\|+\left\|\mathrm{T} \mathrm{T}_{p}-\mathrm{T} \mathrm{T}_{p+1}\right\|\right)}{1+\left\|\mathrm{T}_{p}-\mathrm{x}_{p+1}\right\|+\left\|\mathrm{T}_{p}-\mathrm{T} \mathrm{T}_{p+1}\right\|}, \\
\left\|\mathrm{x}_{p+1}-\mathrm{x}_{p}\right\|, \frac{1}{4} \frac{2\left\|\mathrm{x}_{p+1}-\mathrm{x}_{p+2}\right\|\left(1+\left\|\mathrm{T} \mathrm{x}_{p}-\mathrm{x}_{p}\right\|\right)}{1+\left\|\mathrm{x}_{p}-\mathrm{x}_{p+1}\right\|}, \\
\frac{r}{2} \max \left\{\left\|\mathrm{x}_{p}-\mathrm{x}_{p+1}\right\|,\left\|\mathrm{x}_{p+1}-\mathrm{x}_{p+2}\right\|\right\}
\end{array}\right\} \\
& \leq \frac{\Upsilon}{2} \max \left\{\left\|\mathbf{x}_{p+1}-\mathrm{x}_{p}\right\|,\left\|\mathrm{x}_{p+1}-\mathrm{x}_{p}\right\|,\left\|\mathrm{x}_{p+1}-\mathrm{x}_{p+2}\right\|\right\} \\
& =\frac{r}{2} \max \left\{\left\|\mathrm{x}_{p+1}-\mathrm{x}_{p}\right\|,\left\|\mathrm{x}_{p+1}-\mathrm{x}_{p+2}\right\|\right\} .
\end{aligned}
$$

Consequently, since $\frac{\Upsilon}{2}<1$, we get

$$
\left\|\mathrm{x}_{p+1}-\mathrm{x}_{p+2}\right\| \leq \frac{\Upsilon}{2}\left\|\mathrm{x}_{p+1}-\mathrm{x}_{p}\right\| .
$$

and letting $\kappa=\max \left\{\frac{\Upsilon}{2}, \frac{a \Upsilon}{2}\right\}$, from (2.23) and (2.24) we have

$$
\left\|\mathrm{x}_{p+1}-\mathrm{x}_{p+2}\right\| \leq \kappa\left\|\mathrm{x}_{p+1}-\mathrm{x}_{p}\right\|,
$$


for any $p \in \mathbb{N}$.

Again, letting $\mathrm{x}=\mathrm{x}_{p}$ and $\mathrm{y}=u$ in (2.22), we get

$$
\begin{aligned}
& \left\|\mathrm{Tx}_{p}-\mathrm{T} u\right\| \leq \Upsilon \max \left\{\begin{array}{c}
\left\|\mathrm{x}_{p}-u\right\|, \frac{1}{2} \frac{\left\|\mathrm{x}_{p}-\mathrm{T} \mathrm{x}_{p}\right\|(1+\|u-\mathrm{T} u\|)}{1+\left\|\mathrm{x}_{p}-u\right\|+\left\|\mathrm{x}_{p}-\mathrm{T} u\right\|},\left\|u-\mathrm{Tx}_{p}\right\|, \\
\frac{1}{4} \frac{\|u-\mathrm{T} u\|\left(1+\left\|\mathrm{x}_{p}-\mathrm{T} \mathrm{x}_{p}\right\|\right)}{1+\left\|\mathrm{x}_{p}-u\right\|}, \frac{1}{2}\left\|\mathrm{x}_{p}-\mathrm{T} u\right\|
\end{array}\right\} \\
& \leq \Upsilon\left\|\mathrm{x}_{p}-u\right\|+\frac{\Upsilon}{2} \max \left\{\frac{\left\|\mathrm{x}_{p}-\mathrm{Tx}_{p}\right\|(1+\|u-\mathrm{T} u\|)}{1+\left\|\mathrm{x}_{p}-u\right\|+\left\|\mathrm{x}_{p}-\mathrm{T} u\right\|},\left\|u-\mathrm{Tx}_{p}\right\|\right\}+ \\
& +\frac{\Upsilon}{4} \max \left\{\frac{\|u-T u\|\left(1+\left\|\mathrm{x}_{p}-\mathrm{T} \mathrm{x}_{p}\right\|\right)}{1+\left\|\mathrm{x}_{p}-u\right\|},\left\|\mathrm{x}_{p}-\mathrm{T} u\right\|\right\} \\
& \leq \Upsilon\left\|\mathrm{x}_{p}-u\right\|+\frac{\Upsilon}{2} \max \left\{\frac{\left\|\mathrm{x}_{p}-\mathrm{Tx}_{p}\right\|(1+\|u-\mathrm{T} u\|)}{1+\left\|\mathrm{x}_{p}-u\right\|},\left\|u-\mathrm{Tx}_{p}\right\|\right\}+ \\
& +\frac{\Upsilon}{4} \max \left\{\frac{\|u-\mathrm{T} u\|\left(1+\left\|\mathrm{x}_{p}-\mathrm{Tx}_{p}\right\|\right)}{1+\left\|\mathrm{x}_{p}-u\right\|},\left\|\mathrm{x}_{p}-\mathrm{T} u\right\|\right\} .
\end{aligned}
$$

Moreover, choosing $c_{1}=\Upsilon, c_{2}=\frac{\Upsilon}{2}$ and $\lambda=\frac{\Upsilon}{4}$, for $p$ sufficiently large, we get (2.11) and then, by Theorem 6 it follows that $u$ is a fixed point of $\mathrm{T}$.

\section{References}

[1] I. Gornicki and B.E. Rhoades, A general fixed point theorem for involutions, Indian J. Pure Appl. Math. 27(1) 1996, 13-23.

[2] V. Berinde, Contracţ̧ii generalizate şi aplicaţii , Editura Club Press 22, Baia Mare, 1997.

[3] I.A. Rus, Generalized contractions and applications, Cluj University Press, Cluj-Napoca, 2001.

[4] V. Berinde, Generalized contractions in quasimetric spaces, Seminar on Fixed Point Theory, Preprint no. 3(1993), 3-9.

[5] V. Berinde, Sequences of operators and fixed points in quasimetric spaces , Stud. Univ. "Babeş-Bolyai", Math., 16(4)(1996), 23-27.

[6] I.A. Rus, The theory of a metrical fixed point theorem: theoretical and applicative relevance, Fixed Point Theory, 9(2008), No. 2, 541-559.

[7] E. Karapınar, A fixed point theorem without a Picard operator, Results in Nonlinear Analysis, Volume 4(2021), Issue 3, Pages $127-129$. 